\title{
Dynamic Traffic Control System using RFID Technology: A Systematic Review
}

\author{
Priyanka Nalawade $^{1}$, Prajakta Waghere ${ }^{1}$, Nisha Vanare $^{1}$, Prajakta Kalbhor ${ }^{1}$, A. J. Jadhav ${ }^{2}$ \\ Student, Dept of IT, Rajarshi Shahu College of Engineering, Savitribai Phule Pune University, Pune, India ${ }^{1}$ \\ Professor, Dept of IT, Rajarshi Shahu College of Engineering, Savitribai Phule Pune University, Pune, India ${ }^{2}$
}

\begin{abstract}
Internet of Things (IoT) links the real world objects to the virtual world, and enables every time, everywhere connectivity for anything that can be switched ON/OFF. It constitutes to a world where physical things and human beings, as well as virtual data and environments, interact with each other. Traffic in urban areas is increasing day by day which leads to most critical issues of traffic management. Traffic congestion often causes loss of time, time delay, missed opportunities, etc. To overcome these problems proposed system uses the RFID technology where RFID tag is attached to each vehicle and as and when vehicle reach the traffic junction the RFID reader will read those RFID tags. Depending upon the count of vehicles green passage will be set dynamically and the proposed system provides special privileges for emergency vehicles like police vehicle, ambulance, VIP vehicles, etc. RFID also enforces law against stolen vehicles.
\end{abstract}

Keywords: RFID tag, RFID reader, Ardunio microcontroller, IR sensor.

\section{INTRODUCTION}

Traffic light posts are positioned at the traffic junction. Traffic light set the green passage for specific period of time which is not a complete systematic system as it cannot solve the traffic problems fully. The proposed system will have RFID readers at the traffic junctions that will read all the RFID tags attached to the vehicles coming towards the junction. RFID technology consists following components:

\section{Radio-frequency-identification (RFID)tag:}

RFID tag uses electromagnetic fields to identify and track tags attached to objects automatically. The tags contain electronically saved information. Passive tags gain energy from a nearby RFID reader's interrogating radio waves. Active tags have their own power source such as a battery and may operate at hundreds of meters from the RFID reader.

\section{RFID reader:}

An RFID reader's function is to identify RFID tags. A reader contains a Radio Frequency module, which acts as both a transmitter as well as receiver of radio frequency signals. The transmitter consists of an oscillator to create the carrier frequency. The receiver has a demodulator to extract the returned data and also for processing it contains an amplifier to strengthen the signal. A microprocessor forms the control system, memory to filter and store the data. The data is then ready to be sent to the network.

RFID technology uses digital data within RFID tag, which is made up of integrated circuits which contains a small antenna for transferring information to RFID readers. The majority of RFID tags contain at least an integrated circuit for modulating and demodulating RF signals and an

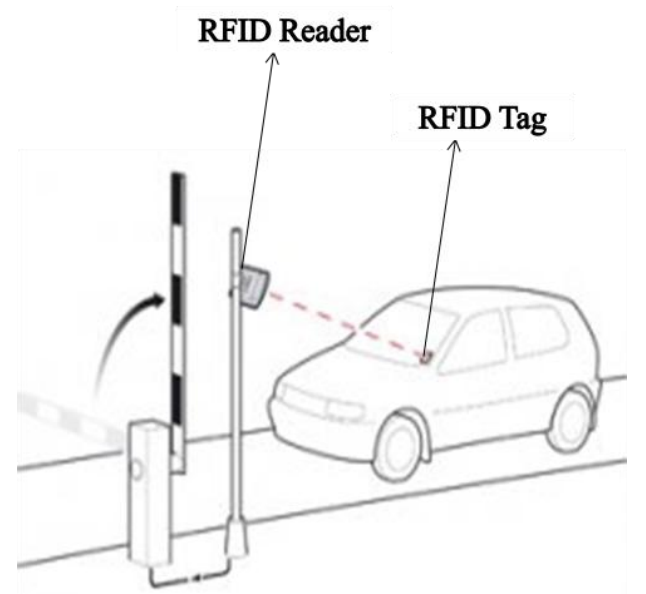

Figure 2.1. Structure of RFID

antenna for transmitting and receiving signals. Frequency ranges differ from low frequencies of 125 to $134 \mathrm{kHz}$ and 140 to $148.5 \mathrm{kHz}$, and high frequencies of 850 to 950 $\mathrm{MHz}$ and 2.4 to $2.5 \mathrm{GHz}$. Wavelengths in the $2.4 \mathrm{GHz}$ range.

\section{EXISTING SYSTEM}

A. Camera [1].

In this particular system, there will be four cameras in one intersection for a junction road. A CPU will be connected with these cameras which will be used for video processing. The RFID will be placed under the road for detection of the car. The hardware's that are used: HD Camera, CPU (For video processing), Microprocessor (For traffic light controlling) and RFID Reader (For vehicle 
Vol. 6, Issue 1, January 2017

detection) which will be besides the road. Then using frame by frame Real-time video analysis through developed algorithm, how much cars are present on the road can be detected. Depending on the number of detected vehicles implementation of a sequential traffic timer system is done. This information will be transmitted to CPU or the central database.

According to this automatic traffic system, the traffic light ON/OFF will depend on the number of vehicles on the road. The CPU will detect each and every car and will count the vehicle number in the road by using the developed algorithm. It will also do the same thing with other side of the road by using another camera. CPU then compares vehicle number on adjacent roads. The road which has more vehicles will get the preference and green light for that road will be set on and red signal will be displayed automatically to the other road. Traffic lights will be connected to the central system and intelligent system will control the traffic light system.

\section{B. WSN [2].}

The work in proposed an approach to integrate Wireless Sensor Network (WSN) in the RFID Reader to implement traffic management system where motion sensors are used to transmitsignal to the RFID reader to enter in the read area when it detects the RFID tag and then RFID Reader reads the contents of RFID tag and pass this information to host via IEEE 802.15.4/Zigbee standard, that reduces the cost and time required by eliminating the wired installation of cable.

Each intersection contains 8 RFID readers. The road is divided into two separate lanes. Each lane has its RFID reader to track the vehicles passing through it. Each intersection point has its separate database to store the information regarding the vehicles that passed from junction. Every vehicle has a RFID enabled device that stores a vehicle identification number (VIN). The task of the intelligent traffic controller is to calculate the time of green signal that is based on the number of vehicle and also solve the problem of Starvation.

\section{Image Processing [3].}

A camera is fixed on polls to overlook the traffic scene. Images received from the video are then analyzed to detect and count number of vehicles. Then depending on the cycle of signal (we have taken it to be 3 minutes), time is allotted to each lane. Generating the current background image based on segmentation results. After separating the objects, we need to determine their edges in the processed image after subtraction. This is done by using an edge detection algorithm. The method finds out edges using the Sobel approximation to the derivative.

It then returns edges at those points where the gradient of the image is the maximum. After finding the edges the next stage is to count the number of objects as determined by the edges. There have been many algorithms recommended for object detection and contour tracing. After finding the edges then count objects as defined by the edges.

\section{GPS system and ZigBee [4].}

This proposed system has three parts. First part contains automatic signal control system. Here, each vehicle is equipped with an RFID tag and reader at traffic junction. When it comes in the range of RFID reader, it will send the signal to the RFID reader which will track how many vehicles have passed through the junction for a specific period of time and determine the traffic congestion volume. Accordingly, it sets the green passage for that particular path. Second part is for the emergency vehicle detection and clearance. Here, each emergency vehicle contains ZigBee transmitter and the ZigBee receiver will be present at the traffic junction. The buzzer will be turned ON when the vehicle is used for emergency. This will send the signal through the ZigBee transmitter to the receiver. It will turn the traffic light to change to green.

Once the ambulance passes through junction, the receiver no longer receives the ZigBee signal and the traffic light is switched to red. RFID and GPS based automatic traffic clearance system for ambulance. The focus of this work is to reduce the delay for the ambulance to reach to the hospital by automatically clearing the traffic lane, before it reaches the traffic signal. The third part is for detection of stolen vehicle. Here, when the RFID reader reads the RFID tag, it compares it to the registered stolen RFIDs. Also GPS can be placed along with the stolen vehicle detection module, so that the exact location of stolen vehicle is known. If a match is found, it sends SMS to the police control room, so that the vehicle is made to stop in the traffic junction and local police can take appropriate action.

\section{PROPOSED SYSTEM}

In urban areas, the traffic is becoming major problem and efforts are taken to resolve the traffic issues. In the proposed system, the traffic light poles are at the center of traffic junction which is controlled by the microcontroller depending on number of vehicles approaching at the junction. The vehicles are equipped with RFID tagand the unique tag and the vehicle owner's information will be registered at the central database. The junction has a RFID reader that reads the tags approaching towards the junction. Depending on the number of vehicles read by the RFID reader, the microcontroller will set the time and manipulate the signal according to the count. The emergency vehicles are equipped with a special identification tag. The emergency vehicles like ambulance, fire brigade, police vehicles, VIP cars etc. will have special RFID tags when such vehicles come at the traffic junction the RFID reader reads these special tags and manipulate particular lane signal to green thus allowing the emergency vehicles pass easily through the junction and thus avoiding wastage of time. The proposed system 
also provides facility in case if the vehicle is stolen. The owner of the car can register the stolen vehicle information with the central database if the tag read by the reader and registered tag maps then the controller will send the message to the owner of the vehicle and also concerned authority. The person who has stolen the vehicle will be punished rule break. The web portal is used to show the records and charts of the traffic management in terms of stolen vehicle, emergency etc.

\section{CONCLUSION}

The previous systems work efficiently to manage to manage the traffic but does not provide quality of service for emergency and stolen vehicles and use additional hardware like ZigBee etc. and complicate the system. The proposed system works effectively and with higher accuracy in managing the traffic at junction with different priorities at different time.

\section{REFERENCES}

[1]. http://archives-ijaet.org/media/6I5-IJAET0511564- DESIGN-ANDSIMULATION-Co

[2]. Chattaraj, S. Chakrabarti, S. Bansal, S. Halder, A. Chandra, "An Intelligent Traffic Control System using RFID", IEEE Potentials, vol. 28, no. 3, pp. 40-43, May-Jun. 2009.

[3]. S. Sharma, A. Pithora, G. Gupta, M. Goel, M. Sinha, "Traffic light priority control for emergency vehicle using RFID", Int. J. Innov. Eng. Technol., vol. 2, no. 2, pp. 363-366, 2013.

[4]. R. Hegde, R. R. Sali, M. S. Indira, "RFID and GPS based automatic lane clearance system for ambulance", Int. J. Adv. Elect. Electron.Eng., vol. 2, no. 3, pp. 102-107, 2013.

[5]. Abdullahi Chowdhury, "Priority based and secured traffic management system for emergency vehicle using IoT", Engineering \& MIS (ICEMIS) International Conference on, pp. 1-6, 2016.

[6]. Rajeshwari Sundar, Santhoshs Hebbar, Varaprasad Golla, B.M.S College of Emgineering "I Implementing Intelligent Traffic Control System for Congestion Control, Ambulance Clearance, and Stolen Vehicle Detection"IEEE Sensors Journal Volume: 15 Issue: 2

[7]. Andrea Zanella, Nicola Bui, et,al "Internet of things for smart cities" IEEE Internet of things journal vol.1, February 2014

[8]. J. Sherly, D. Somasundareswari "INTERNET OF THINGS BASED SMART TRANSPORTATION SYSTEMS" International Research Journal of Engineering and Technology (IRJET) 2015. Ayoub Fikri, El Mehdi Zrihni, Yassine Salih Alj "A Smartphone Based System for Traffic Congestion Control using RFID tags" International Conference on Electrical and Information Technology ICEIT2015 